\title{
Half-sandwich pentamethylcyclopentadienyl group 9 metal complexes of 2-aminopyridyl ligands: Synthesis, spectral and molecular study
}

\author{
MAHESH KALIDASAN ${ }^{\mathrm{a}}$, SCOTT FORBES ${ }^{\mathrm{b}}$, YURIJ MOZHARIVSKYJ ${ }^{\mathrm{b}}$,* and \\ MOHAN RAO KOLLIPARA ${ }^{\mathrm{a}, *}$ \\ ${ }^{a}$ Department of Chemistry, North Eastern Hill University, Shillong 793 022, India \\ ${ }^{b}$ Department of Chemistry and Chemical Biology, McMaster University, Hamilton, L8S 4M1 Canada \\ e-mail: mohanrao59@gmail.com
}

MS received 08 August 2014; revised 27 November 2014; accepted 30 November 2014

\begin{abstract}
Thereaction of $[\mathrm{Cp} * \mathrm{M}(\mu-\mathrm{Cl}) \mathrm{Cl}]_{2}(\mathrm{M}=\mathrm{Rh}$, Ir) with 2-aminopyridyl based ligands lead to the formation of mononuclear neutral complexes of general formula $\left[\mathrm{Cp} * \mathrm{MCl}_{2}(\mathrm{~L})\right]$ \{ where $\mathrm{L} 1=2$-aminopyridine, L2 = 2-amino-3-picoline, L3=2-amino-3-nirtopyridine, and L4=2-amino-3-pyridine carboxyaldehyde $\}$. The complexes have been characterized by FT-IR, UV-Vis, ${ }^{1} \mathrm{H}^{-13} \mathrm{C}$ NMR and mass spectroscopic methods. X-ray crystallographic studies of the complexes have shown typical piano-stool geometry around the metal centre in which 2-aminopyridyl ligand acts as an $\mathrm{N}$-monodentate ligand and the amino functionality is not involved in metal coordination. The intra/intermolecular arrangement is due to hydrogen bonding.
\end{abstract}

Keywords. Rhodium; Iridium; 2-aminopyridine; 2-amino-3-picoline; 2-amino-3-nirtopyridine.

\section{Introduction}

Among various N-containing heterocyclic ligands, 2-aminopyridyl ligand has received much attention because of their potentially ambidentate nature and pharmacological properties. These ligands bind to a metal ion in a variety of bonding modes such as a monodentate $N$-pyridine ligand as well as bidentate ligand and these compounds play an important role in biology. Metal complexes of these ligands have exhibited antibacterial and anti-fungal activity as indicated by recent literature. ${ }^{1-3}$

Some of the transition metal complexes incorporated with 2-aminopyridyl derivatives have been studied extensively in various applications such as potential anticancer agents and as catalysts. For example, 2aminopyridine rhodium(I) complexes have been found to be effective catalysts for the homogeneous hydrogenation of olefins ${ }^{4}$ and 2 -aminopicoline rhodium(I) was useful in olefin or alkyne hydroacylation and in carboncarbon bond activation. ${ }^{5}$ The mononuclear ruthenium complexes of chiral auxiliary ligands, 2-amino-(4R)phenyl-2-oxazoline (amphox), indanyl-2-amino-(4R,5S)2-oxazoline (aminox) are active catalysts for both transfer of hydrogenation and [4+2] cycloaddition chemistry. ${ }^{6}$

Analysis of cell growth inhibition caused by ruthenium complexes of 2-pyridyl and derivatives on panel

\footnotetext{
*For correspondence
}

of tumour cell lines (HeLa, B16, MDA-MB-361, MDAMB-453 and LS-174) revealed low anti-proliferative activity. Replacement of substituted pyridine ligand in complexes such as picolinic acid exhibits better DNA binding affinity and cytotoxicity. ${ }^{7-10}$

Till now, a few reports are available for metal complexes with the 2-aminopyridine ligands, in which it serves as either a monodentate ligand or a bridging ligand. ${ }^{11-13}$ However, there are no reports dealing with half-sandwich $\mathrm{Rh}$ and Ir complexes with these ligands. In the present contribution, we would like to report syntheses of a series of new mononuclear halfsandwich complexes of $\mathrm{Cp} * \mathrm{Rh}(\mathrm{III})$ and $\mathrm{Cp} * \mathrm{Ir}(\mathrm{III})$ with 2-aminopyridine ligand L1, L2, L3 and L4 respectively and their characterization by suitable techniques.

\section{Experimental}

\subsection{Physical methods and materials}

All reagents were purchased from commercial sources and used as received. $\mathrm{RhCl}_{3} \cdot \mathrm{nH}_{2} \mathrm{O}$ and $\mathrm{IrCl}_{3} \cdot \mathrm{nH}_{2} \mathrm{O}$ were purchased from Arora Matthey limited, 2-aminopyridine, 2-amino-3-picoline were obtained from Aldrich and 2-amino-3-nitropyridine, 2-amino-3-pyridine carboxaldehyde were acquired from Merck. The solvents were purified and dried according to standard procedures. ${ }^{14}$ All the experiments were performed under normal conditions. The dinuclear metal precursors 
Table 1. Crystallographic and structure refinement parameters for selected complexes.

\begin{tabular}{|c|c|c|c|c|c|}
\hline & 3 & 4 & 5 & 6 & 8 \\
\hline Chemical formula & $\mathrm{C} 16 \mathrm{H} 23 \mathrm{Cl} 2 \mathrm{~N} 2 \mathrm{Rh}$ & $\mathrm{C} 16 \mathrm{H} 23 \mathrm{Cl} 2 \mathrm{IrN} 2$ & $\mathrm{C} 15 \mathrm{H} 20 \mathrm{Cl} 2 \mathrm{~N} 3 \mathrm{O} 2 \mathrm{Rh}$ & $\mathrm{C} 15 \mathrm{H} 20 \mathrm{Cl} 2 \mathrm{IrN} 3 \mathrm{O} 2$ & $\mathrm{C} 16 \mathrm{H} 21 \mathrm{Cl} 2 \mathrm{IrN} 2 \mathrm{O}$ \\
\hline Formula Mass & 417.17 & 506.46 & 448.15 & 537.44 & 520.45 \\
\hline Crystal system & Monoclinic & Monoclinic & Orthorhombic & Orthorhombic & Monoclinic \\
\hline$a / \AA$ & $11.912(2)$ & $11.908(2)$ & 7.3892(15) & $7.5125(15)$ & $7.5416(15)$ \\
\hline$b / \AA$ & $8.4318(17)$ & $8.4938(17)$ & $15.071(3)$ & $15.018(3)$ & 13.907(3) \\
\hline$c / \AA$ & $17.051(3)$ & 17.194(3) & $31.125(6)$ & $30.912(6)$ & $9.0916(18)$ \\
\hline$\alpha /^{\circ}$ & 90.00 & 90.00 & 90.00 & 90.00 & 90.00 \\
\hline$\beta /{ }^{\circ}$ & $96.14(3)$ & $96.42(3)$ & 90.00 & 90.00 & $113.68(3)$ \\
\hline$\gamma /{ }^{\circ}$ & 90.00 & 90.00 & 90.00 & 90.00 & 90.00 \\
\hline Unit cell volume $/ \AA^{3}$ & $1702.7(6)$ & $1728.1(6)$ & $3466.2(12)$ & $3487.6(12)$ & $873.3(3)$ \\
\hline Temperature/K & $293(2)$ & 293(2) & 293(2) & 293(2) & $293(2)$ \\
\hline Space group & $\mathrm{P} 21 / \mathrm{c}$ & $\mathrm{P} 21 / \mathrm{c}$ & Pbca & Pbca & $\mathrm{P} 21$ \\
\hline$Z$ & 4 & 4 & 8 & 8 & 2 \\
\hline \multirow[t]{3}{*}{ Index ranges } & $-12 \leq h \geq 14$ & $-14 \leq h \geq 14$ & $-8 \leq h \geq 8$ & $-9 \leq h \geq 9$ & $-8 \leq h \geq 8$ \\
\hline & $-10 \leq \mathrm{k} \geq 5$ & $-10 \leq \mathrm{k} \geq 10$ & $-17 \leq \mathrm{k} \geq 17$ & $-18 \leq \mathrm{k} \geq 16$ & $-1 \overline{6} \leq \mathrm{k} \geq 16$ \\
\hline & $-16 \leq l \geq 21$ & $-21 \leq l \geq 11$ & $-36 \leq l \geq 36$ & $-38 \leq l \geq 34$ & $-10 \leq l \geq 10$ \\
\hline$D_{c}\left(\mathrm{~g} \cdot \mathrm{cm}^{-3}\right)$ & 1.627 & 1.947 & 1.718 & 2.047 & 1.979 \\
\hline$\mu\left(\mathrm{mm}^{-1}\right)$ & 1.312 & 8.031 & 1.305 & 7.975 & 7.953 \\
\hline $\mathrm{F}(000)$ & 848 & 976 & 1808 & 2064 & 500 \\
\hline Reflections collected & 6347 & 7223 & 76975 & 11472 & 16390 \\
\hline Independent reflections & $3481[\mathrm{R}(\mathrm{int})=0.0245]$ & $3527[\mathrm{R}(\mathrm{int})=0.0240]$ & $2993,[\mathrm{R}(\mathrm{int})=0.0276]$ & $3559[\mathrm{R}(\mathrm{int})=0.0286]$ & $3559[\mathrm{R}$ (int) $=0.0504]$ \\
\hline Completeness to $\theta=25.00^{\circ}$ & 99.8 & 99.8 & 99.8 & 99.8 & 99.8 \\
\hline Data/restraints/parameters & $3481 / 0 / 197$ & $3527 / 0 / 197$ & $2993 / 0 / 213$ & $3559 / 0 / 213$ & $2998 / 0 / 151$ \\
\hline Goodness-of-fit on $\mathrm{F}^{2}$ & 1.055 & 1.042 & 1.044 & 1.362 & 1.044 \\
\hline Final $\mathrm{R}$ indices $[\mathrm{I}>2 \sigma(\mathrm{I})]^{*}$ & $0.0278, w_{2}=0.0638$ & $0.0278, \mathrm{wR}_{2}=0.0650$ & $0.0206, \mathrm{wR}_{2}=0.0464$ & $0.0499, \mathrm{wR}_{2}=0.0796$ & $0.0243, w_{2}=0.0576$ \\
\hline $\mathrm{R}$ indices (all data) & $0.0357, \mathrm{wR}_{2}=0.0638$ & $0.0341, \mathrm{wR}_{2}=0.0675$ & $0.0295, \mathrm{wR}_{2}=0.0485$ & $0.0576, \mathrm{wR}_{2}=0.0796$ & $0.0271, w_{2}=0.0584$ \\
\hline $\operatorname{Max}, \operatorname{Min} \Delta \rho / \mathrm{e}\left(\AA^{-3}\right)$ & $0.582,-0.771$ & $1.257,-1.205$ & $0.342,-0.280$ & $1.496,-2.877$ & $1.310,-0.757$ \\
\hline
\end{tabular}

* Structures were refined on $F_{0}^{2}: w R_{2}=\left[\Sigma\left[w\left(F_{0}^{2}-F_{\mathrm{c}}^{2}\right)^{2}\right] / \Sigma w\left(F_{0}^{2}\right)^{2}\right]^{1 / 2}$, where $w^{-1}=\left[\Sigma\left(F_{0}^{2}\right)+(a P)^{2}+b P\right]$ and $P=\left[\max \left(F_{0}^{2}, 0\right)+2 F_{\mathrm{c}}^{2}\right] / 3$. 
$[\mathrm{Cp} * \mathrm{M}(\mu-\mathrm{Cl}) \mathrm{Cl}]_{2}(\mathrm{M}=\mathrm{Rh}$ and $\mathrm{Ir})$ were prepared according to literature methods. ${ }^{15-17}$ NMR spectra are recorded on a Bruker Avance II $400 \mathrm{MHz}$. Infrared spectra were recorded as $\mathrm{KBr}$ pellets on a Perkin- Elmer 983 spectrophotometer. Mass spectra were obtained from Water ZQ-4000 mass spectrometer by ESI method in positive mode. Elemental analyses were performed on a Perkin-Elmer-2400 CH/N analyzer. Absorption spectra were obtained at room temperature using a Perkin- Elmer Lambda 25 UV/ visible spectrophotometer.

\subsection{Single-crystal X-ray structures analyses}

The orange crystals of complexes $3,4,5,6,7$ and 8 were obtained by slow diffusion method of hexane diffused into DCM solution of the corresponding complexes. Single crystal X-ray diffraction measurements were carried out on an Xcalibur, Eos, Gemini and STOE IPDSII diffractometer. Single crystals picked up from the samples were analyzed on a STOE IPDSII diffractometer using $\mathrm{MoK} \alpha$ radiation in the whole reciprocal sphere. A numerical absorption correction was based on the crystal shape originally determined by optical face indexing but later optimized against equivalent reflections using the STOE XShape software. Crystal structures were determined and solved using the SHELX software. ${ }^{18,19}$ Crystallographic details are summarized in table 1 and bond lengths and angles were presented table 2. Figure 1 were drawn with ORTEP $-3^{20}$ and figures $2-3$ were drawn with MERCURY. ${ }^{21}$

\subsection{General procedure for the preparation of mononuclear complexes 1 to 8}

A mixture of metal precursor $[\mathrm{Cp} * \mathrm{M}(\mu-\mathrm{Cl}) \mathrm{Cl}]_{2}(0.1$ $\mathrm{mmol}), 2$-aminopyridyl ligand $(0.2 \mathrm{mmol})$ were dissolved in methanol $(20 \mathrm{~mL})$ and stirred at room temperature. Yellow coloured precipitate formed immediately.
Further reaction mixture was stirred for further two hours to complete the reaction The precipitate was washed with methanol, diethyl ether $(3 \times 10 \mathrm{~mL})$ and dried in vacuum.

2.3a $\left[C p^{*} R h C l_{2}(L 1)\right](1)$ : Red orange solid, Yield $60 \mathrm{mg}$ (76\%). IR (KBr, cm $\left.{ }^{-1}\right): 3383(\mathrm{~s}), 3270(\mathrm{~m})$, 3179(m), 2962(w), 1626(s), 1606(w), 1485(m), 1443(m), 1376(w), 1256(w), 1155(w), 1023(m), 842(m), 786(s); ${ }^{1} \mathrm{H}$ NMR $\left(400 \mathrm{MHz}, \mathrm{CDCl}_{3}\right) \delta 8.49\left(\mathrm{~d}, 1 \mathrm{H}, \mathrm{H}_{\mathrm{JJ}}=5\right.$, py ring), $7.25\left(\mathrm{~d}, 1 \mathrm{H}, \mathrm{H}_{\mathrm{JJ}}=4.5\right.$, py ring $), 6.62(\mathrm{t}, 1 \mathrm{H}$, py ring), $6.49\left(\mathrm{t}, 1 \mathrm{H}\right.$, py ring), $6.12\left(\mathrm{~b}, 2 \mathrm{H}, \mathrm{NH}_{2}\right), 1.57$ (s, 15H, Cp* ring); ${ }^{13} \mathrm{C}$ NMR (400 MHz, DMSO-d $\mathrm{d}_{6}+$ $\left.\mathrm{CDCl}_{3}\right) \delta 8.50,87.84,101.21,113.76,135.34,156.82$; ESI-MS: $404.06\left[\mathrm{M}^{+}\right]$peak; UV-Vis \{Acetonitrile, $\left.\lambda_{\max } \mathrm{nm}\left(\varepsilon / 10^{-4} \mathrm{M}^{-1} \mathrm{~cm}^{-1}\right)\right\}:$ 231(0.43), 295(0.12), 401(0.03). Anal. Calc. for $\mathrm{C}_{15} \mathrm{H}_{21} \mathrm{~N}_{2} \mathrm{Cl}_{2} \mathrm{Rh}$ (403.15): C, 44.69; H, 5.25; N, 6.95. Found: C, 44.80; H, 5.42; $\mathrm{N}, 7.01$.

2.3b $\quad[C p * I r C l=(L 1)](2): \quad$ Yellow solid, Yield $80 \mathrm{mg}$ (80\%). IR ( $\left.\mathrm{KBr}, \mathrm{cm}^{-1}\right): 3373(\mathrm{~s}), 3282(\mathrm{~s}), 3183(\mathrm{~m})$, 2968(w), 1623(s), 1607(w), 1488(s), 1433(m), 1377(m), 1253(m), 1157(w), 1123(m), 1053(m), 842(m), 786(s), 612(m); ${ }^{1} \mathrm{H}$ NMR $\left(400 \mathrm{MHz}, \mathrm{CDCl}_{3}\right) \delta 8.48(\mathrm{~d}, 1 \mathrm{H}$, $\mathrm{H}_{\mathrm{JJ}}=4.5$, py ring), $7.20\left(\mathrm{~d}, 1 \mathrm{H}, \mathrm{H}_{\mathrm{JJ}}=4.25\right.$, py ring), $6.60(\mathrm{t}, 1 \mathrm{H}$, py ring), $6.45(\mathrm{t}, 1 \mathrm{H}$, py ring $), 6.10(\mathrm{~b}, 2 \mathrm{H}$, $\mathrm{NH}_{2}$ ), 1.40 (s, 15H, Cp* ring); ${ }^{13} \mathrm{C}$ NMR $(400 \mathrm{MHz}$, DMSO-d $\left.{ }_{6}+\mathrm{CDCl}_{3}\right) \delta 8.20,88.11,101.98,113.01$, 132.86, 154.96; ESI-MS: $493.72\left[\mathrm{M}^{+}\right]$peak; UV-Vis \{Acetonitrile, $\lambda_{\max } \mathrm{nm}\left(\varepsilon / 10^{-4} \mathrm{M}^{-1} \mathrm{~cm}^{-1}\right)$ \}: $231(0.40)$, 296(0.19). Anal. Calc. for $\mathrm{C}_{15} \mathrm{H}_{21} \mathrm{~N}_{2} \mathrm{Cl}_{2} \mathrm{Ir}$ (492.46): C, 36.58; H, 4.30; N, 5.69. Found: C, 36.70; H, 4.45; N, 5.78 .

2.3c $\left[C p^{*} R h C l_{2}(L 2)\right](3):$ Red orange solid, Yield $79 \mathrm{mg}$ (94\%). IR (KBr, cm $\left.{ }^{-1}\right): 3406(\mathrm{~s}), 3276(\mathrm{~s})$, 3183(m), 2916(w), 1615(s), 1595(w), 1474(s), 1455(m), 1377(m), 1253(m), 1303(w), 1272(w), 1205(w), 1086(w),

Table 2. Selected bond lengths $(\AA)$ and angles $\left({ }^{\circ}\right)$.

\begin{tabular}{lccccc}
\hline Complex & $(3)$ & $(4)$ & $(\mathbf{5})$ & $(\mathbf{6})$ & (8) \\
\hline $\mathrm{M}(1)-\mathrm{Cl}(1)$ & $2.450(7)$ & $2.4467(12)$ & $2.4020(7)$ & $2.398(2)$ & $2.370(6)$ \\
$\mathrm{M}(1)-\mathrm{Cl}(2)$ & $2.4036(7)$ & $2.4084(12)$ & $2.4249(7)$ & $2.418(2)$ & $2.448(6)$ \\
$\mathrm{M}(1)-\mathrm{N}(1)$ & $2.168(2)$ & $2.148(4)$ & $2.2043(19)$ & $2.174(6)$ & $2.2153(19)$ \\
$\mathrm{M}(1)-C_{\text {ave }}$ & $2.153(3)$ & $2.154(5)$ & $2.148(2)$ & $2.146(8)$ & $2.244(3)$ \\
$\mathrm{M}(1)-\mathrm{CNT}$ & 1.776 & 1.773 & 1.770 & 1.765 & 1.775 \\
$\mathrm{Cl}(1)-\mathrm{M}(1)-\mathrm{Cl}(2)$ & $90.00(3)$ & $87.45(5)$ & $89.59(3)$ & $87.33(8)$ & $88.57(9)$ \\
$\mathrm{N}(1)-\mathrm{M}(1)-\mathrm{Cl}(1)$ & $93.33(6)$ & $88.32(5)$ & $88.51(5)$ & $87.24(18)$ & $95.15(18)$ \\
$\mathrm{N}(1)-\mathrm{M}(1)-\mathrm{Cl}(2)$ & $89.77(6)$ & $91.76(10)$ & $93.96(5)$ & $92.58(18)$ & $84.46(16)$ \\
\hline
\end{tabular}

CNT represents the centroid of the $\mathrm{Cp} *$ ring; $\mathrm{C}_{\text {ave }}$ represents the average bond distance centroid of the $\mathrm{Cp} *$ ring carbon and metal atom. 


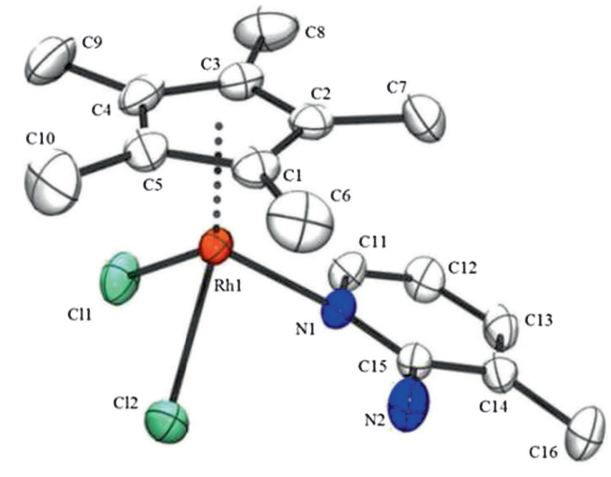

Complex (3)

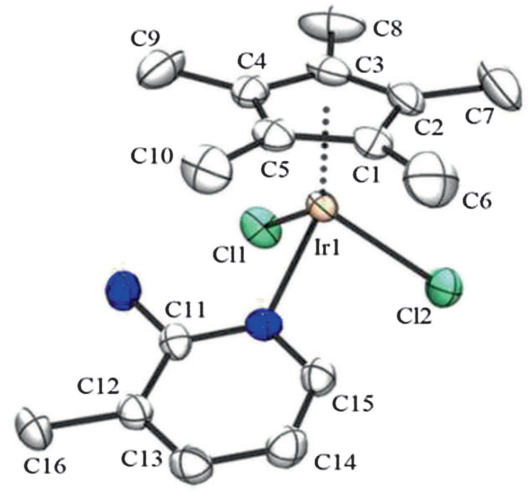

Complex (4)

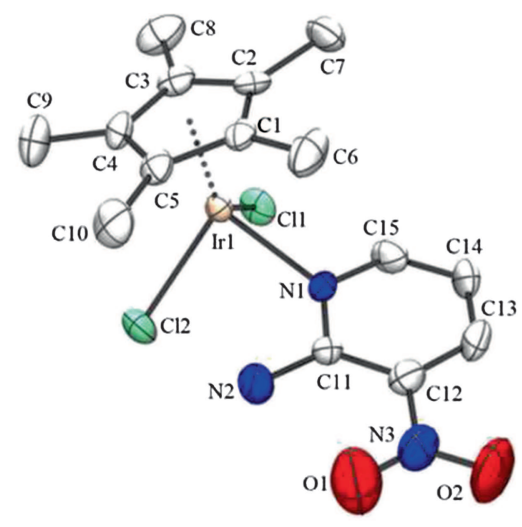

Complex (5)

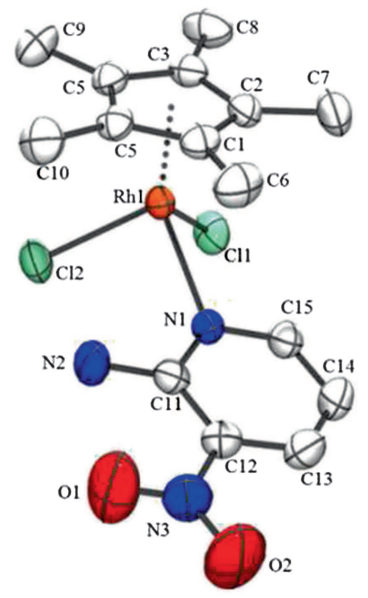

Complex (6)

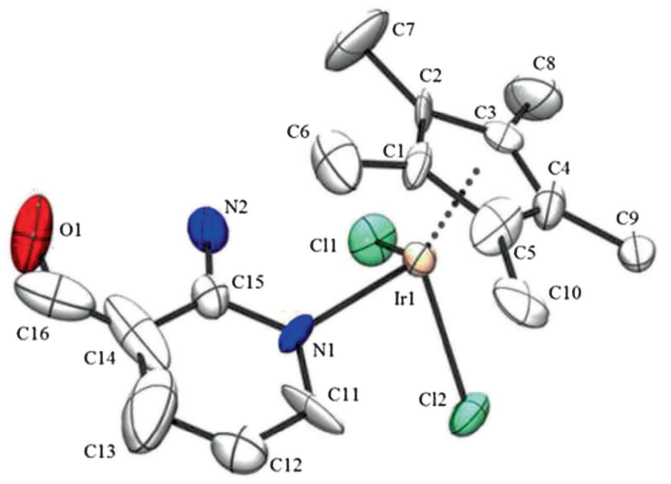

Complex (7)

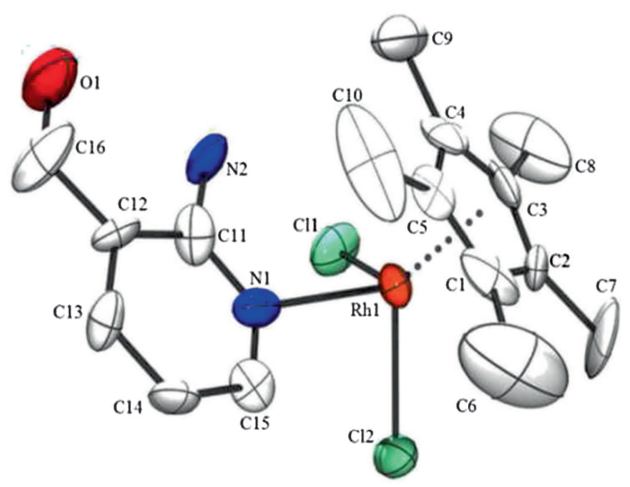

Complex (8)

Figure 1. ORTEP diagram of complexes $\left[\mathrm{Cp}^{*} \mathrm{RhCl}_{2}(\mathrm{~L} 2)\right](\mathbf{3}),\left[\mathrm{Cp} * \mathrm{IrCl}_{2}(\mathrm{~L} 2)\right](\mathbf{4}),\left[\mathrm{Cp}^{*} \mathrm{RhCl}_{2}(\mathrm{~L} 3)\right](\mathbf{5}),\left[\mathrm{Cp}^{*} \mathrm{IrCl}_{2}(\mathrm{~L} 3)\right](\mathbf{6})$ $\left[\mathrm{Cp}^{*} \mathrm{RhCl}_{2}(\mathrm{~L} 4)\right](7)$ and $\left[\mathrm{Cp}^{*} \mathrm{IrCl}_{2}(\mathrm{~L} 4)\right](8)$ with $50 \%$ probability thermal ellipsoids. Hydrogen atoms are omitted for clarity.

1025(m), 789(s), 758(w), 612(m); ${ }^{1} \mathrm{H}$ NMR (400 MHz, $\left.\mathrm{CDCl}_{3}\right) \delta 8.52\left(\mathrm{~d}, 1 \mathrm{H}, \mathrm{H}_{\mathrm{JJ}}=5.25\right.$, py ring), 7.29 (d, $1 \mathrm{H}, \mathrm{H}_{\mathrm{JJ}}=4.75$, py ring), $6.56(\mathrm{t}, 1 \mathrm{H}$, py ring), 6.14 (b, $2 \mathrm{H}, \mathrm{NH}_{2}$ ), 2.12 (s, 3H, $\left.\mathrm{CH}_{3}\right), 1.57$ (s, 15H, Cp* ring); ${ }^{13} \mathrm{C}$ NMR (400 MHz, DMSO- $\left.d_{6}+\mathrm{CDCl}_{3}\right) \delta 8.49$, 88.96, 99.03, 114.24, 135.65, 153.32; ESI-MS: 418.10 $\left[\mathrm{M}^{+}\right.$] peak; UV-Vis \{Acetonitrile, $\lambda_{\max } \mathrm{nm}\left(\varepsilon / 10^{-4} \mathrm{M}^{-1}\right.$ $\left.\left.\mathrm{cm}^{-1}\right)\right\}$ : 230(0.74), 297(0.26), 405(0.06). Anal. Calc. for $\mathrm{C}_{16} \mathrm{H}_{23} \mathrm{~N}_{2} \mathrm{Cl}_{2} \mathrm{Rh}$ (417.18): C, 46.06; H, 4.30; N, 6.71. Found: C, 46.25; H, 4.43; N, 6.89 .

2.3d $\left[C p^{*} \operatorname{IrCl}_{2}(\mathrm{L2})\right](4): \quad$ Yellow solid, Yield $92 \mathrm{mg}$ (92\%). IR (KBr, cm $\left.{ }^{-1}\right)$ : 3381(s), 3278(s), 3187(m), 2972(w), 1627(s), 1601(w), 1473(s), 1452(s), 1381(m), 1301(w), 1261(w), 1199(w), 1083(w), 1035(m), 782(s), 757(w); ${ }^{1} \mathrm{H}$ NMR (400 MHz, $\left.\mathrm{CDCl}_{3}\right) \delta 8.50(\mathrm{~d}, 1 \mathrm{H}$, $\mathrm{H}_{\mathrm{JJ}}=5.25$, py ring), $7.27\left(\mathrm{~d}, 1 \mathrm{H}, \mathrm{H}_{\mathrm{JJ}}=4.50\right.$, py ring), $6.53\left(\mathrm{t}, 1 \mathrm{H}\right.$, py ring), $6.13\left(\mathrm{~b}, 2 \mathrm{H}, \mathrm{NH}_{2}\right), 2.11$ (s, $\left.3 \mathrm{H}, \mathrm{CH}_{3}\right), 1.58$ (s, 15H, Cp* ring); ${ }^{13} \mathrm{C} \mathrm{NMR}(400$ $\left.\mathrm{MHz}, \mathrm{DMSO}-d_{6}+\mathrm{CDCl}_{3}\right) \delta 8.21,89.65,99.59,115.07$,
138.13, 156.37; ESI-MS: $507.87\left[\mathrm{M}^{+}\right]$peak; UV-Vis \{Acetonitrile, $\lambda_{\max } \mathrm{nm}\left(\varepsilon / 10^{-4} \mathrm{M}^{-1} \mathrm{~cm}^{-1}\right)$ \}: 228(0.84), 299(0.26). Anal. Calc. for $\mathrm{C}_{16} \mathrm{H}_{23} \mathrm{~N}_{2} \mathrm{Cl}_{2} \mathrm{Ir}$ (506.49): C, 37.94; H, 4.58; N, 5.53. Found: C, 38.12; H, 4.64; N, 5.70 .

2.3e $\left[\mathrm{Cp}^{*} \mathrm{RhCl}_{2}(\mathrm{L3})\right](5)$ : Red orange solid, Yield $80 \mathrm{mg}$ (89\%). IR (KBr, cm $\left.{ }^{-1}\right): 3380(\mathrm{~s}), 3218(\mathrm{~s})$, 3182(m), 3135(s), 2965(w), 2913(m), 1627(s), 1584(m), 1560(m), 1507(m), 1466(w), 1378(w), 1355(m), 1318(s), 1240(s), 1109(s), 1028(m), 891(w), 760(m), 613(s); ${ }^{1} \mathrm{H}$ NMR $\left(400 \mathrm{MHz}, \mathrm{CDCl}_{3}\right) \delta 8.46\left(\mathrm{~d}, 1 \mathrm{H}, \mathrm{H}_{\mathrm{JJ}}=4.75\right.$, py ring), $7.20\left(\mathrm{~d}, 1 \mathrm{H}, \mathrm{H}_{\mathrm{JJ}}=4.50\right.$, py ring), $6.72(\mathrm{t}$, $1 \mathrm{H}$, Py ring), 6.31 (b, 2H, $\left.\mathrm{NH}_{2}\right), 1.56\left(\mathrm{~s}, 15 \mathrm{H}, \mathrm{Cp}^{*}\right.$ ring); ${ }^{13} \mathrm{C}$ NMR (400 MHz, DMSO- $\left.d_{6}+\mathrm{CDCl}_{3}\right) \delta 8.52$, 78.22, 98.66, 112.16, 134.47, 155.61; ESI-MS: 449.21 $\left[\mathrm{M}^{+}\right]$peak; UV-Vis \{Acetonitrile, $\lambda_{\max } \mathrm{nm}\left(\varepsilon / 10^{-4}\right.$ $\left.\left.\mathrm{M}^{-1} \mathrm{~cm}^{-1}\right)\right\}$ : 262(0.68), 388(0.60). Anal. Calc. for $\mathrm{C}_{15} \mathrm{H}_{20} \mathrm{~N}_{3} \mathrm{Cl}_{2} \mathrm{O}_{2} \mathrm{Rh}$ (448.15): C, 40.20; H, 4.50; N, 9.38. Found: C, 40.42; H, 4.66; N, 9.50. 


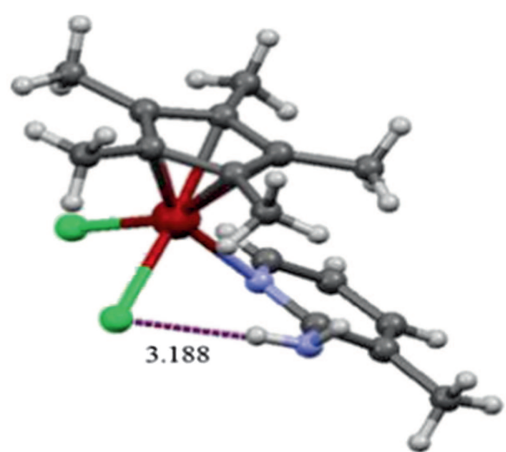

Complex (3)

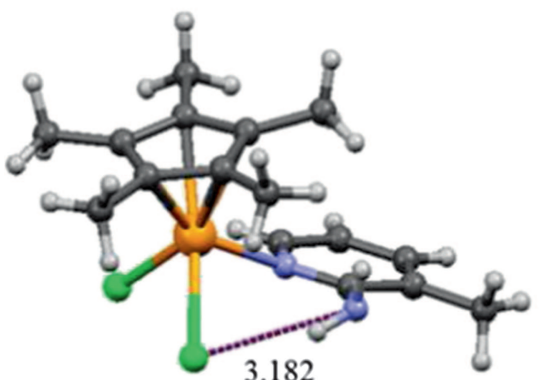

Complex (4)

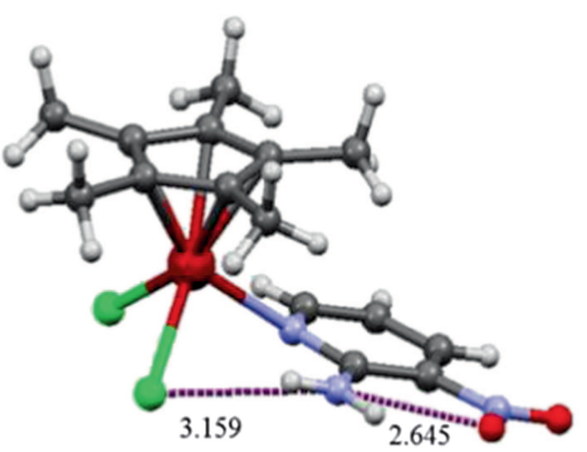

Complex (5)

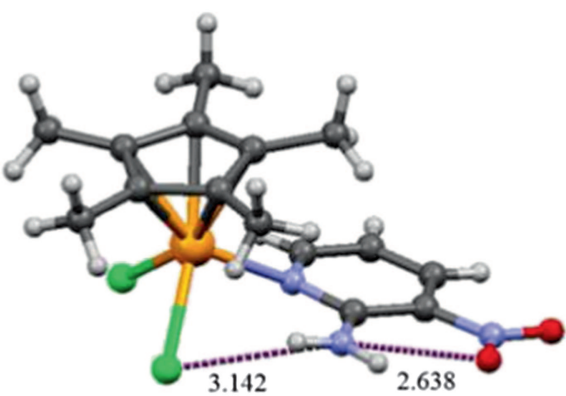

Complex (6)

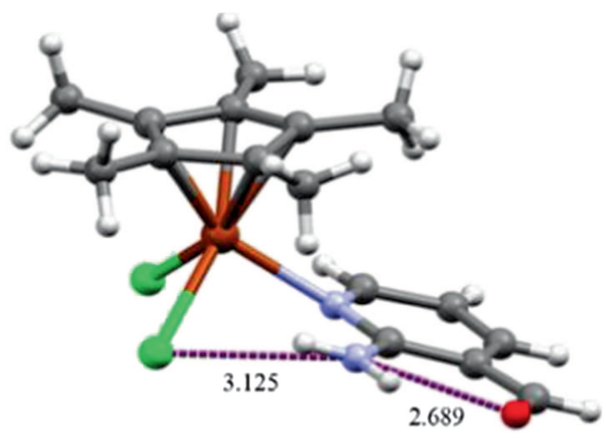

Complex (7)

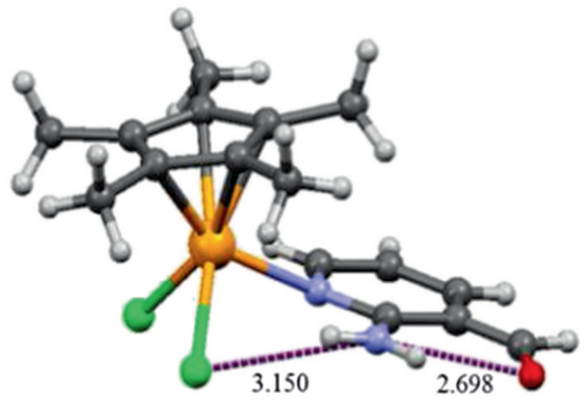

Complex (8)

Figure 2. View of H-bonding in metal complexes.

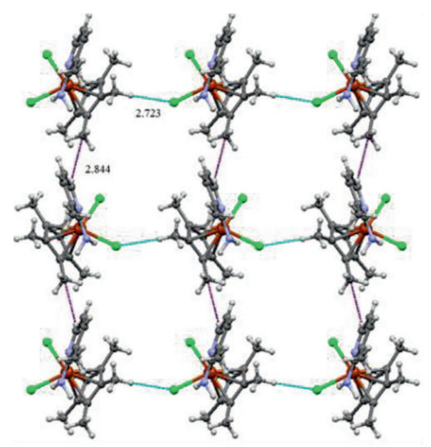

(a)

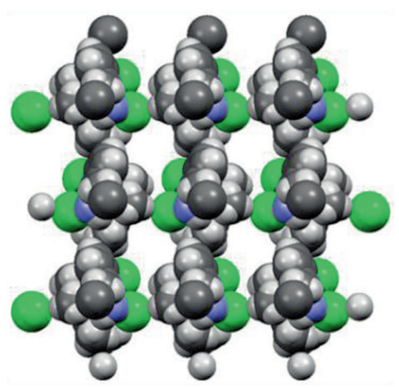

(d)

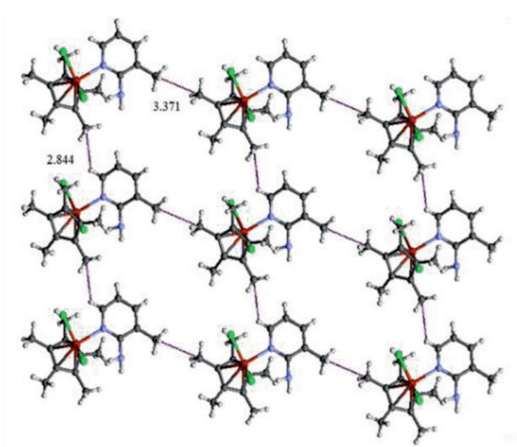

(b)

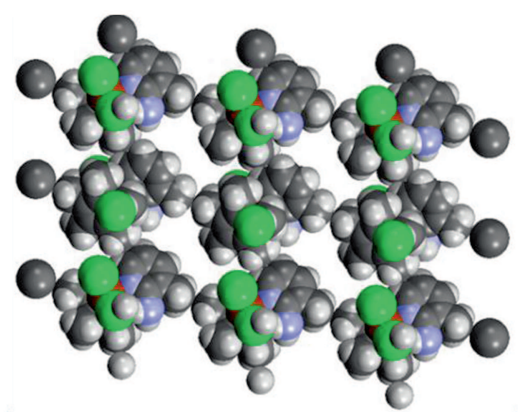

(e)

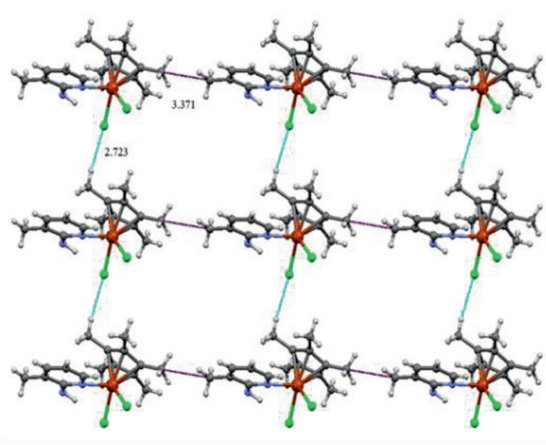

(c)

Figure 3. Self-assembly into discrete network architectures of complex 3; (a) N-H...Cl and CH... $\pi$ interaction along a axis; (b) $\mathrm{CH} . . . \pi$ and $\pi \ldots \pi$ interaction along $\mathrm{b}$ axis; (c) $\mathrm{N}-\mathrm{H} . . . \mathrm{Cl}$ and $\pi \ldots \pi$ interactions along c axis; (d) (e) (f) Space filled models representing weak interactions. 
2.3f $\left[\mathrm{Cp}^{*} \mathrm{IrCl}_{2}(\mathrm{L3})\right](6): \quad$ Yellow solid, Yield $90 \mathrm{mg}$ (82\%). IR (KBr, cm $\left.{ }^{-1}\right)$ : 3386(s), 3222(m), 3186(m), 3140(m), 2967(w), 2919(w), 1629(s), 1582(m), 1559(m), 1510(m), 1445(m), 1384(m), 1353(m), 1317(s), 1240(s), 1109(s), 1037(m), 828(w), 758(m), 611(m); ${ }^{1} \mathrm{H}$ NMR $\left(400 \mathrm{MHz}, \mathrm{CDCl}_{3}\right) \delta 12.12(\mathrm{~s}, 1 \mathrm{H}, \mathrm{CHO}), 8.50(\mathrm{~d}, 1 \mathrm{H}$, $\mathrm{H}_{\mathrm{JJ}}=5.25$, py ring), $7.26\left(\mathrm{~d}, 1 \mathrm{H}, \mathrm{H}_{\mathrm{JJ}}=4.75\right.$, py ring), $6.96\left(\mathrm{t}, 1 \mathrm{H}\right.$, py ring), $6.29\left(\mathrm{~b}, 2 \mathrm{H}, \mathrm{NH}_{2}\right), 1.58$ (s, $15 \mathrm{H}, \mathrm{Cp}^{*}$ ring); ${ }^{13} \mathrm{C}$ NMR (400 MHz, DMSO- $d_{6}+$ $\left.\mathrm{CDCl}_{3}\right) \delta$ 8.18, 77.90, 92.09, 112.20, 134.44, 155.52; ESI-MS: $538.79\left[\mathrm{M}^{+}\right]$peak; UV-Vis $\left\{\right.$Acetonitrile, $\lambda_{\max }$ $\left.\mathrm{nm}\left(\varepsilon / 10^{-4} \mathrm{M}^{-1} \mathrm{~cm}^{-1}\right)\right\}$ : 259(0.97), 380(0.68). Anal. Calc. for $\mathrm{C}_{15} \mathrm{H}_{20} \mathrm{~N}_{3} \mathrm{Cl}_{2} \mathrm{O}_{2} \mathrm{Ir}$ (537.46): C, 33.52; H, 3.75; N, 7.82. Found: C, 33.67; H, 3.86; N, 7.99.

2.3g $\left[C p^{*} R h C l_{2}(L 4)\right](7):$ Red orange solid, Yield $70 \mathrm{mg}(81 \%)$. IR (KBr, cm $\left.{ }^{-1}\right): 3360(\mathrm{~s}), 3242(\mathrm{~s})$, 3212(w), 2965(w), 2917(w), 1664(s), 1613(s), 1562(s), 1456(m), 1381(m), 1259(m), 1197(m), 1124(s), 1026(m), 909(w), 771(s), 673(s), 611(m); ${ }^{1} \mathrm{H}$ NMR (400 MHz, $\left.\mathrm{CDCl}_{3}\right) \delta 12.12(\mathrm{~s}, 1 \mathrm{H}, \mathrm{CHO}), 8.46\left(\mathrm{~d}, 1 \mathrm{H}, \mathrm{H}_{\mathrm{JJ}}=4.50\right.$, py ring), $7.20\left(\mathrm{t}, 1 \mathrm{H}\right.$, py ring), $6.95\left(\mathrm{~d}, 1 \mathrm{H}, \mathrm{H}_{\mathrm{JJ}}=4.75\right.$, py ring), 6.30 (b, $2 \mathrm{H}, \mathrm{NH}_{2}$ ), 1.58 (s, $15 \mathrm{H}, \mathrm{Cp}^{*}$ ring); ${ }^{13} \mathrm{C}$ NMR (400 MHz, DMSO-d $\left.{ }_{6}+\mathrm{CDCl}_{3}\right) \delta 8.52,78.74$, 98.62, 111.92, 144.16, 154.52, 192.91; ESI-MS: 432.25 $\left[\mathrm{M}^{+}\right.$] peak; UV-Vis \{Acetonitrile, $\lambda_{\max } \mathrm{nm}\left(\varepsilon / 10^{-4} \mathrm{M}^{-1}\right.$ $\left.\left.\mathrm{cm}^{-1}\right)\right\}$ : 259(0.77), 349(0.65), 407(0.20). Anal. Calc. for $\mathrm{C}_{16} \mathrm{H}_{21} \mathrm{~N}_{2} \mathrm{Cl}_{2} \mathrm{ORh}$ (431.16): $\mathrm{C}, 44.57 ; \mathrm{H}, 4.91 ; \mathrm{N}$, 6.50. Found: C, 44.68; H, 5.06; N, 6.67.

2.3h $\quad\left[C p^{*} \operatorname{IrCl}_{2}(L 4)\right](8): \quad$ Yellow solid, Yield $88 \mathrm{mg}$ (85\%). IR ( $\left.\mathrm{KBr}, \mathrm{cm}^{-1}\right): 3362(\mathrm{~s}), 3255(\mathrm{~s}), 2967(\mathrm{w})$, 2918(w), 1667(s), 1614(s), 1565(s), 1453(m), 1404(w), 1381(m), 1258(m), 1196(m), 1123(s), 1034(m), 909(w), 768(m), 674(m), 612(m); $\left.{ }^{1} \mathrm{H} \mathrm{NMR} \mathrm{(400} \mathrm{MHz,} \mathrm{CDCl}_{3}\right) \delta$ $12.17(\mathrm{~s}, 1 \mathrm{H}, \mathrm{CHO}), 8.45\left(\mathrm{~d}, 1 \mathrm{H}, \mathrm{H}_{\mathrm{JJ}}=5.0\right.$, py ring), 7.24(t, $1 \mathrm{H}$, py ring $), 6.98\left(\mathrm{~d}, 1 \mathrm{H}, \mathrm{H}_{\mathrm{JJ}}=4.75\right.$, py ring), 6.31 (b, 2H, $\mathrm{NH}_{2}$ ), 1.56 (s, 15H, $\mathrm{Cp}^{*}$ ring); ${ }^{13} \mathrm{C}$ NMR $\left(400 \mathrm{MHz}, \mathrm{DMSO}-\mathrm{d}_{6}+\mathrm{CDCl}_{3}\right) \delta 8.32,78.53$, 97.92, 114.64, 145.67, 155.27, 192.71; ESI-MS: 521.91 $\left[\mathrm{M}^{+}\right]$peak; UV-Vis \{Acetonitrile, $\lambda_{\max } \mathrm{nm}\left(\varepsilon / 10^{-4}\right.$ $\left.\mathrm{M}^{-1} \mathrm{~cm}^{-1}\right)$ \}: 259(0.41), 346(0.47). Anal. Calc. for $\mathrm{C}_{16} \mathrm{H}_{21} \mathrm{~N}_{2} \mathrm{Cl}_{2} \mathrm{OIr}$ (520.47): C, 36.92; H, 4.07; N, 5.38. Found: C, 37.15; H, 4.13; N, 5.45.

\section{Results and Discussion}

\subsection{Synthesis of the complexes}

A series of eight new neutral half-sandwich $\mathrm{Rh}$ and Ir complexes have been synthesized from the reaction of $\left[\mathrm{Cp}^{*} \mathrm{M}(\mu-\mathrm{Cl}) \mathrm{Cl}\right]_{2}(\mathrm{M}=\mathrm{Rh}, \mathrm{Ir})$ with $1: 2$ molar equivalents of 2-aminopyridyl ligand (L) in methanol (Scheme 1). These complexes were isolated in very good yield and purified by recrystallization method. All these complexes are red orange/ yellow crystalline solids, resulting as non-hygroscopic, air-stable. They are highly soluble in water and DMSO but they are sparingly soluble in DCM, acetone, acetonitrile and chloroform. All these complexes are fully characterized by IR, ${ }^{1} \mathrm{H} \&{ }^{13} \mathrm{C}$ NMR, mass and electronic spectroscopy. Our efforts to make disubstituted mononuclear cationic complexes of type $\left[\mathrm{Cp} * \mathrm{MCl}(\mathrm{L})_{2}\right]^{+}$with 1:4 molar equivalents of 2-aminopyridyl ligands were unsuccessful.

\subsection{Spectral studies of the complexes}

IR spectra of metal complexes show bands around at $1625,1600 \mathrm{~cm}^{-1}$ and $1565 \mathrm{~cm}^{-1}$, typical for the

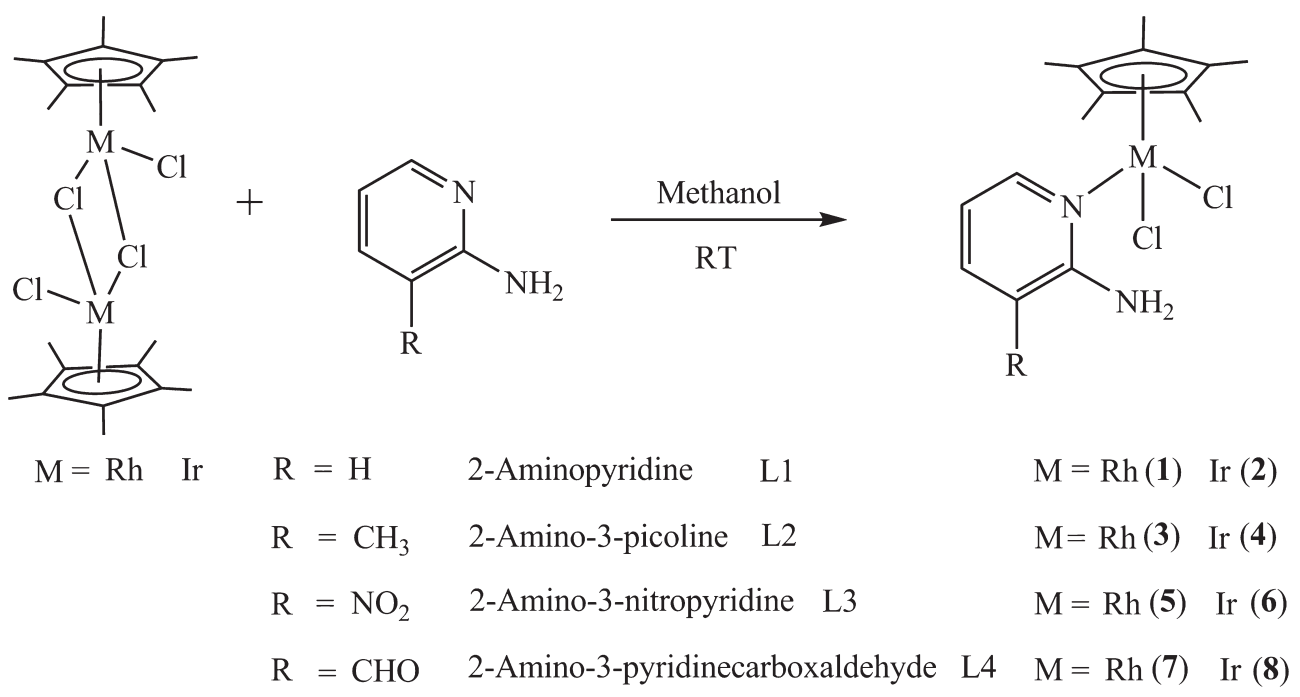

Scheme 1. Syntheses of $\mathrm{Cp} * \mathrm{Rh} / \mathrm{Cp} * \mathrm{Ir}$ metal compounds. 
pyridine skeleton and the out of plane peak at 786 $\mathrm{cm}^{-1}$. 2-aminopyridyl ligands have two nitrogen atoms and both of them can coordinate to the metal ion. When amino nitrogen atom is involved in coordination a strong lower frequency observed in $\mathrm{NH}_{2}$ stretching vibration $\left(\Delta=150-200 \mathrm{~cm}^{-1}\right){ }^{22}$ Since there is no shift in $\mathrm{NH}_{2}$ stretching vibrations, amino nitrogen of 2-aminopyridyl ligands does not involve in coordination in these complexes. The ring breathing mode observed at $983 \sim 898 \mathrm{~cm}^{-1}$ for 2-aminopyridyl ligands, is observed at $1025 \sim 1010 \mathrm{~cm}^{-1}$ for the complexes. This mode is known to be very sensitive to coordination of pyridine from the ring nitrogen atom and the wave number increases with the strength of the coordination bond. Thus, it is concluded that ring nitrogen is involved in the coordination to the metal in the complexes studied. ${ }^{23}$

${ }^{1} \mathrm{H}$ NMR spectra of all metal complexes, the pyridyl ring protons have shown the signal around 8.5-6.5 ppm to corresponding protons and $\mathrm{NH}_{2}$ group signal shown as broad signal around $6.2 \mathrm{ppm}$. Complexes 2 and $\mathbf{3}$ have shown $\mathrm{CH}_{3}$ protons at $2.1 \mathrm{ppm}$ and complexes $\mathbf{7}$ and $\mathbf{8}$ have shown proton of $\mathrm{CHO}$ group at $12.1 \mathrm{ppm}$ The peaks for 2-aminopyridyl ligand protons are observed at higher frequencies in complexes relative to the free ligands, confirming coordination to the $\mathrm{Rh} / \mathrm{Ir}$ via the pyridine-N atom ${ }^{24}$ Moreover all the complexes have displayed a singlet at $\delta 1.58$ and $\delta 1.53 \mathrm{ppm}$ due to the $\mathrm{Cp}^{*}$ ligand, which is a slight change in the position of proton signal in comparison to those observed in the starting material of $\left[\mathrm{Cp} * \mathrm{MCl}_{2}\right]_{2}$ dimer. ${ }^{25}$

The ${ }^{13} \mathrm{C}$ NMR spectra of all complexes exhibited five signal in the region at $85.60-155.90 \mathrm{ppm}$ for pyridyl ring carbons and sharp single peak at $8.50 \mathrm{ppm}$ that corresponding to $\mathrm{Cp}^{*}$ methyl carbon. The complexes 3, 4, 7 and 8 showed single peak at $27.60 \mathrm{ppm}$ and 192.50 ppm for methyl and CHO group carbon of corresponding complexes respectively. These signals are shifted to downfield compared to starting metal precursor and free ligand which indicated the formation of metal complexes.

The mass spectra of all the complexes show their $\left[\mathrm{M}^{+}\right]$molecular ion peaks as per theoretically expected values and supporting well the formation of complexes. The rhodium complexes display their $\left[\mathrm{M}^{+}\right]$molecular ion peaks at $\mathrm{m} / \mathrm{z}$ 404.06, 418.10, 449.21, 432.25 respectively. The iridium complexes show their $\left[\mathrm{M}^{+}\right]$molecular ion peaks at $\mathrm{m} / \mathrm{z} 493.72,507.87,538.79,521.91$ respectively.

UV- Visible spectra were recorded in acetonitrile at $10^{-4} \mathrm{M}$ concentration in the range $200-650 \mathrm{~nm}$ for the all the complexes and shown in figure 4 . Spectroscopic

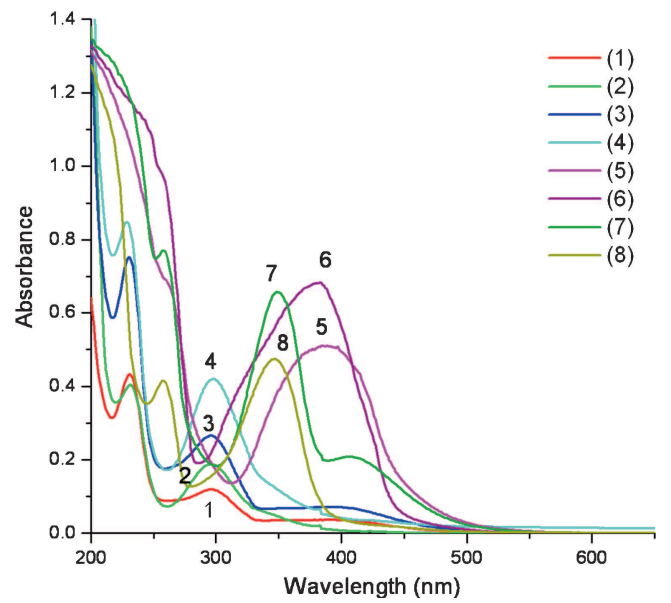

Figure 4. UV- Visible spectra in acetonitrile at $10^{-4} \mathrm{M}$ concentration in the range $200-650 \mathrm{~nm}$ for the complexes.

data for complexes are presented in table 3. The low spin $\mathrm{d}^{6}$ - configuration of the mononuclear complexes provides filled orbitals of proper symmetry, which can interact with low lying $\pi^{*}$ orbitals of the 2aminopyridyl ligand. One should therefore expect a band attributable to the MLCT $\left(\mathrm{t}_{2 \mathrm{~g}}-\pi^{*}\right)$ transition of the electronic spectra, where the transition energy of these bands vary with the nature of the ligands acting as $\pi$-acceptors. The presence of an electron donating group $\left(\mathrm{CH}_{3}\right)$ in the 2-aminopyridyl ligand decreases of energy of transition, causing red shift in MLCT transition maxima, while an electron withdrawing group $\left(\mathrm{NO}_{2}, \mathrm{CHO}\right)$ increases the transition energy ${ }^{26}$ The UV-visible spectra of all the complexes reveal an intense ligand-localized or intra-ligand $\pi-\pi *$ transition in the UV region and a metal to ligand charge transfer (MLCT) $d \pi(M)-\pi *(L)$ band in the visible region. All the spectra show a high intense absorption band around 23-300 nm, assigned to a $\pi-\pi *$ transition, while the less intense absorption band at approximately 360, 390, $405 \mathrm{~nm}$ which are assigned to metal to ligand charge transfers ${ }^{27,28}$

Table 3. UV-Vis data of the complexes.

\begin{tabular}{llll}
\hline Complexes & \multicolumn{3}{c}{$\lambda$ max $/ \mathrm{nm}\left(\varepsilon / 10^{-4} \mathrm{M}^{-1} \mathrm{~cm}^{-1}\right)$} \\
\hline $\mathbf{1}$ & $231(0.43)$ & $295(0.12)$ & $401(0.03)$ \\
$\mathbf{2}$ & $231(0.40)$ & $296(0.19)$ & \\
$\mathbf{3}$ & $230(0.74)$ & $297(0.26)$ & $405(0.06)$ \\
$\mathbf{4}$ & $228(0.84)$ & $299(0.26)$ & \\
$\mathbf{5}$ & $262(0.68)$ & $388(0.60)$ & \\
$\mathbf{6}$ & $259(0.97)$ & $380(0.68)$ & \\
$\mathbf{7}$ & $259(0.77)$ & $349(0.65)$ & $407(0.20)$ \\
$\mathbf{8}$ & $259(0.41)$ & $346(0.47)$ & \\
\hline
\end{tabular}




\subsection{Molecular structural studies}

The molecular structures of the mononuclear complexes 3, 4, 5, 6 and 8 have been established by singlecrystal X-ray structure analysis. Complexes 3, 4 and $\mathbf{8}$ are crystallized in the monoclinic space group $P 2_{1} / \mathrm{c}$ and $P 21$, and complexes 5 and $\mathbf{6}$ in the orthorhombic space group Pbca. The crystal structure of complex 7 could not be properly solved due to the low number of observed reflections so we have presented only the structure and composition of the molecule. Crystallographic and structure refinement parameters for complexes are shown in table 1 and selected bond distances and bond angles are shown in table 2. The $\mathrm{H}$ bonding interactions of metal complexes are shown in table 4. All the complexes display in three-legged piano stool geometry with the metal centre coordinated by the aromatic ligand $\mathrm{Cp}^{*}$ and the other three sites to be coordinated by two chloride atoms and monodentate 2-aminopyridyl ligand. The metal atom of all the complexes is in pseudo octahedral arrangement (figure 1).

In the rhodium compounds, the average distance between the metal atom and the $\mathrm{C}$-atoms of the $\mathrm{Cp}^{*}$ is $2.153 \AA$ (3), 2.148 $\AA$ (5) and $2.163 \AA$ (7). The distance between the $\mathrm{Rh}$ atom and the $\mathrm{Cp}$ * ring centroid ranges from 1.765 to $1.812 \AA$ while iridium compounds. The average distance between the metal atom and the $\mathrm{C}$ atoms of the $\mathrm{Cp}^{*}$ is $2.154 \AA$ (4) and $2.146 \AA$ (8), and the distance between the Ir atom and the $\mathrm{Cp}^{*}$ ring centroid range around $1.770 \AA$. These bond distances are almost identical and same as those for other monodentate pyridine-based binuclear $\mathrm{Cp} * \mathrm{Rh} / \mathrm{Cp}$ *Ir complexes. ${ }^{29-31}$

The average distance of $\mathrm{Rh}-\mathrm{Cl}$ bond length is 2.427 $\AA$ (3), $2.413 \AA$ (5) and $2.399 \AA$ (7) almost identical to the average distance of $\mathrm{Ir}-\mathrm{Cl}$ bond length is $2.427 \AA$ (4) and $2.408 \AA(8)$, while $\mathrm{Rh}-\mathrm{N}$ bond distance is $2.168(2)$ $\AA$ 3, 2.204(19) $\AA 5$ and 2.248(15) $\AA 7$ shorter than Ir$\mathrm{N}$ bond distance is 2.148(4) $\AA$ (4) and 2.174(6) $\AA$ (8). The $\mathrm{M}-\mathrm{Cl}$ distance does not change significantly but the $\mathrm{M}-\mathrm{N}$ distance changes drastically depend on the substitution at the 2-aminopyridyl ligand. ${ }^{32,33}$ This could be explained by the difference in electron density at the metal centre caused by the additional of various groups on the 2-aminopyridyl ring. The better donating ability of the methyl substituted 2-aminopyridyl ligand leads to a somewhat weaker $\mathrm{M}-\mathrm{N}$ interaction in $\mathbf{4}$ relative to that in 7 . The bond angles of $\mathrm{M}$ with ligand and chloride atom are around $87-95^{\circ}$ which are normal and close to $90^{\circ}$ that is evidence for 'piano stool' geometry.

In crystal packing, hydrogen bonding and aromaticaromatic interactions play important role. In all the complexes, aromatic-aromatic interactions as well as hydrogen bonding are expected to influence crystal packing. ${ }^{34,35}$ We have observed that two different types of hydrogen bonding pattern (intra/inter) in metal complexes depending on the nature of substituent of 2aminopyridyl components. These hydrogen bonding

Table 4. H- bonding interaction of complexes.

\begin{tabular}{llllll}
\hline Complex & Donor-H....Acceptor & D-H & H...A & D...A & D-H...A \\
\hline $\mathbf{3}$ & $\mathrm{N}(2)-\mathrm{H}(2) \ldots \mathrm{Cl}(2) \mathrm{i}$ & 0.86 & 2.30 & 3.188 & 153 \\
& $\mathrm{C}(6)-\mathrm{H}(6 \mathrm{~B}) \ldots \mathrm{Cl}(2) \mathrm{ii}$ & 0.96 & 2.72 & 3.673 & 173 \\
$\mathbf{4}$ & $\mathrm{N}(2)-\mathrm{H}(2) \ldots \mathrm{Cl}(2)$ & 0.86 & 2.35 & 3.182 & 162 \\
$\mathbf{5}$ & $\mathrm{C}(6)-\mathrm{H}(6 \mathrm{~B}) \ldots \mathrm{Cl}(2) \mathrm{iii}$ & 0.96 & 2.76 & 3.696 & 164 \\
& $\mathrm{~N}(2)-\mathrm{H}(2 \mathrm{~A}) \ldots \mathrm{Cl}(2)$ & 0.86 & 2.37 & 3.1586 & 152 \\
& $\mathrm{~N}(2)-\mathrm{H}(2 \mathrm{~B}) \ldots \mathrm{O}(1)$ & 0.86 & 2.04 & 2.6454 & 127 \\
& $\mathrm{~N}(2)-\mathrm{H}(2 \mathrm{~B}) \ldots \mathrm{Cl}(1) \mathrm{iv}$ & 0.86 & 2.59 & 3.2527 & 135 \\
$\mathbf{6}$ & $\mathrm{C}(8)-\mathrm{H}(8 \mathrm{~B}) \ldots \mathrm{Cl}(2) \mathrm{v}$ & 0.96 & 2.83 & 3.6209 & 141 \\
& $\mathrm{~N}(2)-\mathrm{H}(1) \ldots \mathrm{Cl}(2)$ & 0.97 & 2.23 & 3.142 & 155 \\
& $\mathrm{~N}(2)-\mathrm{H}(2) \ldots \mathrm{O}(2)$ & 0.72 & 2.14 & 2.645 & 128 \\
& $\mathrm{~N}(2)-\mathrm{H}(2) \ldots \mathrm{Cl}(1) \mathrm{vi}$ & 0.72 & 2.69 & 3.259 & 138 \\
$\mathbf{8}$ & $\mathrm{C}(8)-\mathrm{H}(8 \mathrm{~A}) \ldots \mathrm{Cl}(2) \mathrm{vii}$ & 0.96 & 2.81 & 3.669 & 149 \\
& $\mathrm{~N}(2)-\mathrm{H}(2 \mathrm{~A}) \ldots \mathrm{O}(1)$ & 0.86 & 2.09 & 2.6984 & 127 \\
& $\mathrm{~N}(2)-\mathrm{H}(2 \mathrm{~B}) \ldots \mathrm{Cl}(1)$ & 0.86 & 2.35 & 3.1500 & 155 \\
& $\mathrm{C}(12)-\mathrm{H}(12) \ldots \mathrm{O}(1) \mathrm{viii}$ & 0.93 & 2.53 & 3.3014 & 140 \\
& $\mathrm{C}(16)-\mathrm{H}(16) \ldots \mathrm{Cl}(1) x \mathrm{i}$ & 0.93 & 2.60 & 3.3927 & 144 \\
\end{tabular}

Symmetry transformations used to generate equivalent atoms: $\mathrm{i}=1$ $\mathrm{x}, 2-\mathrm{y},-\mathrm{z}$, ii $=\mathrm{x},-1+\mathrm{y}, \mathrm{z}$, iii $=\mathrm{x},-1+\mathrm{y}, \mathrm{z}$, iv $=3 / 2-\mathrm{x},-1 / 2+\mathrm{y}, \mathrm{z}, \mathrm{v}=$ $3 / 2-x, 1 / 2+y, z$, vi $=3 / 2-x, 1 / 2+y, z$, vii $=3 / 2-x,-1 / 2+y, z$, viii $=2-x,-$ $1 / 2+\mathrm{y}, 3-\mathrm{z}, \mathrm{xi}=1+\mathrm{x}, \mathrm{y}, 1+\mathrm{z}$. 
distances between $\mathrm{N}-\mathrm{H}-\mathrm{Cl}$ are in the range of 3.142$3.188 \AA$ and distances between $\mathrm{N}-\mathrm{H}-\mathrm{O}$ are in the range of 2.638-2.698 $\AA$ (figure 2). The main reason for the discrepancy in hydrogen bonding distance results from the increased acidity (increased donor ability) of the 2aminopyridyl ligand. ${ }^{36-38}$

In addition, weak interaction studies reveal the presence of various $\mathrm{CH} . . . \mathrm{Cl}, \mathrm{CH} \ldots \pi, \pi \ldots \pi$ interactions in the solid state. Interestingly, in complex $\mathbf{3}$ it is observed that distances between $\mathrm{CH} . . \mathrm{Cl}, \mathrm{CH} \ldots \pi, \pi \ldots \pi$ are $2.71-2.84,2.58-2.83$ and 3.83-3.84 $\AA$, respectively and result in a grid 2D sheet Complex 4 shows distances between $\mathrm{CH} . . . \mathrm{Cl}, \pi \ldots \pi$ as $2.71-2.84,3.83-3.84$ $\AA$, respectively and result in a grid $2 \mathrm{D}$ sheet but without $\mathrm{CH} . . . \pi$ interaction. These interactions may be called van der Waals interactions, since the lengths are significantly less than the sum of the van der Waals radii (2.8 and $3.1 \AA$, respectively). ${ }^{39}$ (figure 3 ).

\section{Conclusion}

In summary, $\mathrm{Cp} * \mathrm{Rh}$ and $\mathrm{Cp} * \mathrm{Ir}$ complexes containing with 2-aminopyridyl ligand have been synthesized successfully. All these complexes are fully characterized by spectroscopic methods and confirmed by XRD techniques. X-ray analyses established the coordination of nitrogen atoms to the metal centres as monodentate ligand. Moreover, all the metal complexes containing 2aminopyridine were found to form inter/intramolecular hydrogen bonds such as $\mathrm{N}-\mathrm{H}-\mathrm{Cl}$ and also $\mathrm{CH}$...Cl, $\mathrm{CH} . . . \pi$, and $\pi \ldots \pi$ interactions.

\section{Supplementary Information}

CCDC numbers 1018330 (3), 101831 (4), 1018332 (5), 1018333 (6), 1018334 (7), 1018335 (8) contain the supplementary crystallographic data for the compounds. These data can be obtained free of charge via www.ccdc.cam.ac.uk/data_request/cif, by e-mailing data_request@ccdc.cam.ac.uk, or by contacting The Cambridge Crystallographic Data Centre, 12, Union Road, Cambridge CB2 1EZ, UK; fax: +44 1223 336033.

\section{Acknowledgements}

Mahesh K thanks University Grants Commission (UGC), New Delhi for providing financial assistance in the form of University Fellowship (UGC-RFSMS). K. M. Rao gratefully acknowledges financial support from Council of Scientific and Industrial Research
(CSIR) New Delhi, through the Research grants No. 01(2493)/11/EMR-II.

\section{References}

1. Liang J, Wang Y, Yu J, Li Y and Xu R 2003 Chem. Comm. 882

2. Yip J H K, Suwarno and Vittal J J 2000 Inorg. Chem. 39 3537

3. Munzurglu O and Geckil H 2004 Arch. Environ. Contam. Toxicol. 43203

4. Zuber M, Szuberla W A and Pruchnik F 1986 J. Mol. Catal. $\mathbf{3 8} 309$

5. Jun C H 2004 Chem. Soc. Rev. 33610

6. Cabeza J A, Silva I, Rio I, Gossage R A, Mendez L M and Miguel D 2007 J. Organomet. Chem. 692 4346

7. Antonarakis E S and Emandi A 2010 Cancer Chemother. Pharmacol. 661

8. Galanski M, Arion V B, Jakupec M A and Keppler B K 2003 Curr. Pharm. Des. 92078

9. Vacca A, Bruno M, Boccarelli A, Coluccia M, Ribatti D, Bergamo A, Garbisa S, Sartor L and Sava G 2002 Br. J. Cancer 86993

10. Gligorijevic N, Arandelovic S, Filipovic L, Jakovljevic K, Jankovic R, Sipka S G, Ivanovic I, Radulovic S and Tesic Z L 2012 J. Inorg. Biochem. 10853

11. Garralda M A, Hernandez R, Ibarlucea L, Pinilla E, Torres M R and Zarandona M 2007 Organometallics 26 5369

12. Cabeza J A 2002 Eur. J. Inorg. Chem. 1559

13. Sakai K, Akiyama N and Mizota M 2003 Acta Cryst. E59 459

14. Perrin D D and Armarego W L F 1996 In Purification of Laboratory Chemicals $4^{\text {th }}$ edition (London: Butterworths-Heinemann)

15. Kang J W, Moseley K and Maitlis P M 1969 J. Am. Chem. Soc. 915970

16. Ball R G, Graham W A G, Heinekey D M, Hoyano J K, McMaster A D, Mattson B M and Michel S T 1990 Inorg. Chem. 292023

17. White C, Yates A and Maitlis P M 1992 Inorg. Synth. 29 228

18. STOE X-Shape; STOE \& Cie Gmb H: Darmstadt, Germany, 2004

19. Sheldrick G M, SHELXS-97 and SHELXL-97, University of Gottingen, Gottingen, Germany (1999)

20. Farrugia L J 1997 J. Appl. Cryst. 30565

21. Poel H V D, Koten G V and Vrieze K 1980 Inorg. Chem. 191145

22. Arnaudov M G, Ivanova B B and Dinkov S 2005 Vibr. Spectrosc. 37145

23. Bakiler M, Maslov I V and Akyuz S 1999 J. Mol. Struct. 47583

24. Sahay A N, Pandey D S and Walawalkar M G $2000 \mathrm{~J}$. Organomet. Chem. $\mathbf{6 1 3} 250$

25. Prasad K T, Therrien B and Rao K M $2010 \mathrm{~J}$. Organomet. Chem. $\mathbf{6 9 5} 226$

26. Lavallee D K, Baughman M D and Phillips M P 1977 J. Am. Chem. Soc. 99718

27. Pal S, Das D, Sinha C and Kennard C H L 2001 Inorg. Chim. Acta 31321

28. Kobler E M and Meyer T J 1982 Inorg. Chem. 21 3967 
29. Prasad K T, Therrien B and Rao K M 2008 J. Organomet. Chem. 6933049

30. Govindaswamy P, Canivet J, Therrien B, Suss-Fink G, Stepnicka P and Ludvik J 2007 J. Organomet. Chem. 6923664

31. Lara S B, Habtemariam A, Clarkson G J and Sadlar P J 2011 Eur. J. Inorg. Chem. 3257

32. Kohl G, Pritzkow H and Enders M 2008 Eur. J. Inorg. Chem. 4230

33. Cermak J, Zadny J, Krupkova A, Lopatova K, Vlachova A, Thi T H N, Sauliova J, Sykora J and C1sarova I 2007 J. Organomet. Chem. 6921557
34. Furrer M A, Furrer J and Therrien B 2012 Organometallics 313149

35. Han Y F, Jia W G, Lin Y J and Jin G X $2008 \mathrm{~J}$. Organomet. Chem. 693546

36. Zhu Y Y, Yi H P, Li C, Jiang X K and Li Z 2008 Cryst. Growth Des. 81294

37. Zhu S, Nieger M, Daniels J, Felder T, Kossev I, Schmidt T, Sokolowski M, Vögtle F and Schalley C A 2009 Chem. Eur. J. 155040

38. Etter M C 1990 Acc. Chem. Res. 23120

39. Zheng X J, Li L C, Gao S and Jin L P 2004 Polyhedron 231257 